Louisiana State University

LSU Digital Commons

Faculty Publications

Department of Physics \& Astronomy

$1-1-1998$

\title{
Frequency-resolved time-gated high-order harmonics
}

\author{
Carlo Altucci \\ Lunds Tekniska Högskola \\ Christian Delfin \\ Lunds Tekniska Högskola \\ Lena Roos \\ Lunds Tekniska Högskola \\ Mette B. Gaarde \\ Niels Bohr Institutet \\ Anne L'Huillier \\ Lunds Tekniska Högskola
}

See next page for additional authors

Follow this and additional works at: https://digitalcommons.Isu.edu/physics_astronomy_pubs

\section{Recommended Citation}

Altucci, C., Delfin, C., Roos, L., Gaarde, M., L'Huillier, A., Mercer, I., Starczewski, T., \& Wahlström, C. (1998). Frequency-resolved time-gated high-order harmonics. Physical Review A - Atomic, Molecular, and Optical Physics, 58 (5), 3934-3941. https://doi.org/10.1103/PhysRevA.58.3934

This Article is brought to you for free and open access by the Department of Physics \& Astronomy at LSU Digital Commons. It has been accepted for inclusion in Faculty Publications by an authorized administrator of LSU Digital Commons. For more information, please contact ir@lsu.edu. 


\section{Authors}

Carlo Altucci, Christian Delfin, Lena Roos, Mette B. Gaarde, Anne L’Huillier, Ian Mercer, Tomas Starczewski, and C. G. Wahlström 


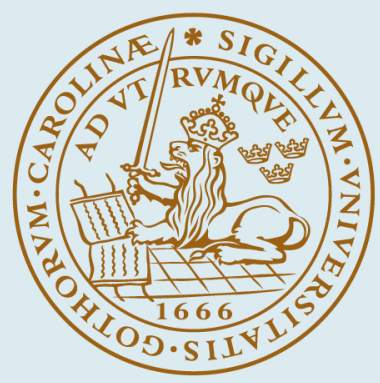

\title{
LUND UNIVERSITY
}

\section{Frequency-resolved time-gated high-order harmonics}

\author{
Altucci, C; Delfin, C; Roos, L; Gaarde, Mette; L'Huillier, Anne; Mercer, I; Starczewski, Tomas; \\ Wahlström, Claes-Göran \\ Published in: \\ Physical Review A (Atomic, Molecular and Optical Physics)
}

DOI:

10.1103/PhysRevA.58.3934

1998

Link to publication

Citation for published version (APA):

Altucci, C., Delfin, C., Roos, L., Gaarde, M., L'Huillier, A., Mercer, I., Starczewski, T., \& Wahlström, C-G. (1998).

Frequency-resolved time-gated high-order harmonics. Physical Review A (Atomic, Molecular and Optical

Physics), 58(5), 3934-3941. https://doi.org/10.1103/PhysRevA.58.3934

Total number of authors:

8

\footnotetext{
General rights

Unless other specific re-use rights are stated the following general rights apply:

Copyright and moral rights for the publications made accessible in the public portal are retained by the authors and/or other copyright owners and it is a condition of accessing publications that users recognise and abide by the legal requirements associated with these rights.

- Users may download and print one copy of any publication from the public portal for the purpose of private study or research.

- You may not further distribute the material or use it for any profit-making activity or commercial gain

- You may freely distribute the URL identifying the publication in the public portal

Read more about Creative commons licenses: https://creativecommons.org/licenses/

Take down policy

If you believe that this document breaches copyright please contact us providing details, and we will remove

access to the work immediately and investigate your claim.
} 


\title{
Frequency-resolved time-gated high-order harmonics
}

\author{
Carlo Altucci, ${ }^{1,2}$ Christian Delfin, ${ }^{1}$ Lena Roos, ${ }^{1}$ Mette B. Gaarde, ${ }^{3}$ Anne L'Huillier, ${ }^{1}$ Ian Mercer,,${ }^{4, *}$ \\ Tomas Starczewski, ${ }^{1}$ and C.-G. Wahlström ${ }^{1}$ \\ ${ }^{1}$ Department of Physics, Lund Institute of Technology, P.O. Box 118, S-221 00 Lund, Sweden \\ ${ }^{2}$ INFM, Sezione Napoli, Naples, Italy \\ ${ }^{3}$ Orsted Laboratory, Niels Bohr Institute, 2100 Copenhagen, Denmark \\ ${ }^{4}$ Department of Chemistry and Biochemistry, Imperial College, South Kensington, London, United Kingdom
}

(Received 12 May 1998)

\begin{abstract}
We report measurements of high-order harmonic spectra obtained with a 800-nm 150-fs laser pulse with a time-varying degree of ellipticity. The modulation of the polarization in time is achieved by using birefringent optics and self-phase modulation in a glass plate. We can create one or two temporal gates of a few femtoseconds width, during which the polarization is linear and harmonic emission is efficient. The harmonic spectra observed experimentally demonstrate that harmonics generated with linear polarization are frequency chirped. The values measured experimentally are consistent with theoretical predictions based on the strong field approximation. [S1050-2947(98)05511-5]
\end{abstract}

PACS number(s): 32.80.Rm, 42.65.Ky

\section{INTRODUCTION}

The generation of high-order harmonics in gases with strong laser fields has been studied for several years and considerable progress has been made in the understanding of the physics involved. This progress is, to a large extent, due to the development of the quasiclassical interpretation [1] and its quantum-mechanical formulations [2,3]. In this approach, an electron initially in the ground state of an atom and exposed to an intense, low-frequency, linearly polarized, electromagnetic field, first tunnels through the barrier formed by the Coulomb and the laser field. When the laser field changes sign, the electron may be driven back towards the atomic core and recombine to the ground state, giving rise to emission of high-energy harmonic photons.

This simple description of harmonic generation processes has led to new ideas and proposals.

(i) One of these ideas is that harmonic generation is extremely sensitive to the laser degree of ellipticity, since a small amount of laser ellipticity can modify the electron trajectory enough to make the electron miss the atomic core when it returns, thus preventing recombination and harmonic generation. Indeed, several experiments have demonstrated the high sensitivity of harmonic generation to the laser degree of ellipticity [4]. This provides a way to control the harmonic emission by modulating the laser degree of ellipticity. Mercer et al. [5] have demonstrated experimentally how the harmonic spatial mode could be manipulated by varying in space the laser degree of ellipticity. Corkum and co-workers $[6,7]$ have proposed to modulate in time the laser degree of ellipticity, using two cross-polarized pulses with slightly different frequencies, so as to restrict the harmonic emission to a short time at the maximum of the combined laser pulse, during which the total field becomes linearly polarized.

\footnotetext{
*Present address: Lawrence National Livermore Laboratory, L440, Livermore, CA 94450.
}

(ii) The phase of the harmonic components depends strongly on the laser peak intensity. This is because the phase of the harmonic emission relative to the fundamental is locked to the quasiclassical action, i.e., the accumulated phase of the electronic wave function, at the time when it recombines [8,9]. Calculations [9] predict that the harmonic phase varies rapidly, and almost linearly, with intensity. This phase variation with the laser intensity affects the phasematching conditions, as well as the spatial and temporal properties of the harmonics [10]. In particular, the variation of the laser intensity during the pulse leads to a significant phase modulation in time, and hence to a frequency modulation (chirp) of the harmonic pulse.

(iii) Another fascinating idea is the possibility to generate "attosecond" pulses (of duration a few hundreds of attoseconds), using harmonics. There are essentially two types of proposals, those that concern harmonics in the plateau $[6,11,12]$, and those that concern one or a few harmonics in the cutoff [13-16]. The first types of proposals are based on the observation that the harmonic spectra consist of a plateau with peaks equally spaced in frequency and with nearly equal amplitude. Farkas and Toth, and Harris et al. [11] proposed that, by filtering out some harmonics from the plateau, one could obtain a train of intense ultrashort pulses of attosecond duration. Antoine et al. [12] showed theoretically that, though it does not turn out to be true on the level of individual atoms, the emission from a macroscopic medium of atoms consists indeed of trains of attosecond pulses. As proposed by Corkum and co-workers [6], by combining this idea and the temporal modulation of the laser degree of ellipticity described above, it might be possible to generate a single attosecond pulse (see also $[17,18])$. In the second type of proposal $[13-16,18]$, one simply uses very short fundamental pulses (below $25 \mathrm{fs}$ ) and high laser intensities (inducing high ionization rates) to generate very short harmonic pulses. Further compression can be achieved by compensating the dynamically induced frequency modulation (chirp) of the harmonics $[10,13]$. 


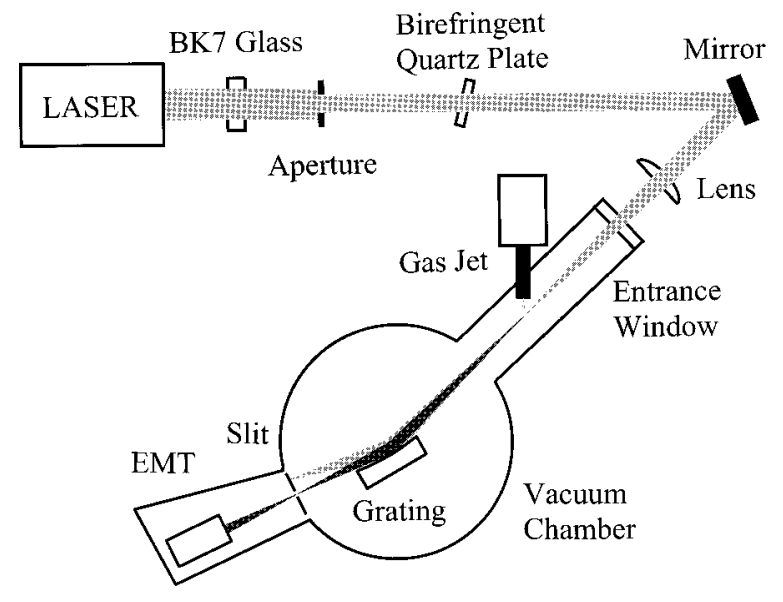

FIG. 1. Experimental setup.

In the present work, we develop a simple technique to modulate in time the laser degree of ellipticity, using birefringent optics and chirped laser pulses. This leads to a total laser field, the polarization of which varies in time. Depending on the configuration, we can open one or two "gates" in time, during which the laser degree of ellipticity is practically linear and harmonic emission is possible. Using one gate, we generate ultrashort harmonic pulses, the duration of which is estimated to be about 5 fs. Using two gates, we show in addition that harmonics are frequency chirped, thus confirming the prediction of the semiclassical model. The information that we get is similar to the one obtained with "frequency-resolved optical-gating" type of instruments in the optical domain [19]. By opening two gates in time, we obtain two distinct peaks in the frequency domain (for each harmonic). The significant frequency separation between these two peaks cannot be simply explained by a chirp due to the laser only. This demonstrates that the harmonic generation process intrinsically leads to harmonic pulses exhibiting a frequency modulation.

The paper is organized as follows: In Sec. II, we describe in detail the experimental method, with emphasis on the technique employed to vary the polarization of the fundamental field. The experimental results obtained are presented in Sec. III. We discuss them and conclude in Sec. IV.

\section{EXPERIMENTAL METHOD}

\section{A. Experimental setup}

The experimental setup is schematically shown in Fig. 1. The laser used in the present experiment is the terawatt titanium sapphire laser of the Lund High-Power Laser Facility [20], operated at a wavelength of $800 \mathrm{~nm}$, with a 110-fs pulse duration and up to $150 \mathrm{~mJ}$ energy. To manipulate in time the degree of polarization of the incident light, as explained in detail below, we use two optical flats: a $10-\mathrm{mm}$ thick glass (BK7) window and a 2.6-mm-thick birefringent quartz plate. The $50-\mathrm{mm}$-diameter beam is apertured down to a diameter of $20 \mathrm{~mm}$ or less and focused by a $f=2 \mathrm{~m}$ lens into a vacuum chamber. Harmonics are generated in a 1-mmlong, pulsed jet of neon atoms. They are separated with a grazing-incidence toroidal grating and detected by an electron multiplier (EMT) placed behind a $200-\mu \mathrm{m}$ slit. Typical recorded spectra extend from the 17 th harmonic to the $61 \mathrm{st}$ harmonic, with a cutoff located around the 51st harmonic. The intensity in the interaction region is estimated to be in the range $(2-5) \times 10^{14} \mathrm{~W} / \mathrm{cm}^{2}$, depending on the measurements.

\section{B. Glass plate}

The glass (BK7) plate introduces a self-phase modulation $\Phi(t)=n_{2} k L I(t)=B f(t)$, proportional to the laser intensity $I(t)=I_{0} f(t)$ and to the thickness $L=10 \mathrm{~mm}$ of the plate, characterized by a nonlinear refractive index $n_{2}=4$ $\times 10^{-16} \mathrm{~W}^{-1} \mathrm{~cm}^{2} . k=2 \pi / \lambda$ is the laser wave vector. The laser peak intensity on the plate $I_{0}$ is varied between 6 and $9 \times 10^{10} \mathrm{~W} / \mathrm{cm}^{2}$ and is almost uniform over the small aperture used. In most of the measurements presented, $I_{0}=7.5$ $\times 10^{10} \mathrm{~W} / \mathrm{cm}^{2}$. In this case, the $B$ integral through the glass plate $\left(B=n_{2} k L I_{0}\right)$ is equal to 2.4 .

The induced frequency shift, defined as

$$
\Delta \omega(t)=\omega(t)-\omega_{0}=-\partial \Phi(t) / \partial t
$$

increases approximately linearly at the middle of the pulse. (The minus sign comes from our convention for the phase, defined as $\int^{t}\left[\omega_{0}-\omega\left(t^{\prime}\right)\right] d t^{\prime}$.) Consequently, the chirp induced by the glass plate is positive; i.e., the rising edge of the pulse is shifted to the red, and the falling edge to the blue.

\section{Quartz plate}

The orientation of the birefringent quartz plate can be described by three different angles, $\alpha, \beta$, and $\gamma$. The angle $\alpha$ denotes the angle between the optic axis of the plate and the (horizontal) laser polarization direction. In addition, the plate is rotated by an angle $\gamma$ around the vertical axis, and by an angle $\beta$ around the optic axis. When $\alpha=45^{\circ}$, the laser electric field is split into two perpendicularly polarized components of equal amplitude, along the ordinary and the extraordinary axes. The difference in refractive indices induces a time delay between the two components. The refractive index of the extraordinary component depends on the angle $\gamma$. To find the refractive index as a function of $\gamma$, an index ellipsoid is constructed, in this case with one axis equal to $n_{e}$, corresponding to the direction of the optic axis and the other two axes equal to $n_{o}$. The refractive index is found at the intersection of the ellipsoid with a plane perpendicular to the direction of propagation which contains the center of the ellipsoid. The intersection plane is an ellipse with semiaxes corresponding to $n_{o}$ and $n_{e}(\gamma)$ :

$$
\frac{1}{n_{e}(\gamma)^{2}}=\frac{\sin ^{2}(\gamma)}{n_{o}^{2}}+\frac{\cos ^{2}(\gamma)}{n_{e}^{2}}
$$

Varying $\gamma$ allows us to choose the time difference between the two components. In the experiments presented below, we use a quartz-plate thickness of $2.6 \mathrm{~mm}$ and $\gamma=20^{\circ}$, leading to a time delay for the extraordinary component compared to the ordinary of approximately $73 \mathrm{fs}$ at a laser wavelength of $800 \mathrm{~nm}$. To determine precisely the time delay, we have taken into account the fact that the two waves propagate different distances in air as well as in quartz due to refraction. We have also included the (small) effect of the different group velocities for the two pulses. 
Finally, the quartz plate can be tilted around its optic axis by an angle $\beta$. This alters the effective thickness of the plate without changing the refractive index of the extraordinary wave and makes it possible to accurately control the relative phase shift, $\Phi_{0}$, between the two components.

\section{Total $B$ integral}

In order to calculate the entire phase change encountered by the beam through the optics, it is necessary to consider, in addition to self-phase modulation in the glass plate, other contributions to dispersion (see Fig. 1): The air path between the laser and the vacuum chamber $\left(n_{2}=4.7\right.$ $\times 10^{-19} \mathrm{~W}^{-1} \mathrm{~cm}^{2}, L \approx 2 \mathrm{~m}$ ), the quartz plate, the focusing lens, and the entrance window. All of these three optical components are made in fused silica $\left(n_{2}=2.8\right.$ $\times 10^{-16} \mathrm{~W}^{-1} \mathrm{~cm}^{2}$ ), and with respective thickness $2.6,5$, and $5 \mathrm{~mm}$. For the last two components, since they are placed after the birefringent plate, where the beam is split into two orthogonally polarized time-delayed components, one has to consider, not only self-phase modulation, induced by one component on itself, but also cross-phase modulation, induced by one component on the other [21]. We estimate the contribution of all these components (including that of the air path) to the $B$ integral to be about 1 so that the total $B$ integral is slightly above 3 . (In the following calculation, we use $B=3$.)

The temporal variations of the phases $\Phi_{x}$ and $\Phi_{y}$ of the two cross-polarized pulses after passage through all the optics are shown in Fig. 2 (thin solid and dashed lines). We approximate the laser temporal profile before the quartz plate as a Gaussian distribution with a 110 -fs full width at half maximum. The total pulse (after passage through the quartz plate) has a rather flat temporal shape with a maximum at center and a 150-fs width at half maximum. The origin of time is chosen to be located between the two pulses, at the maximum of the total pulse. The phase shift $\Phi_{0}$ (modulus $\pi$ ) induced by the quartz plate between the two pulses is equal to 0 in (a) and $\pi / 2$ in (b).

\section{E. Laser chirp}

In Fig. 3 we show the wavelength shift

$$
\Delta \lambda(t)=-\frac{\lambda^{2}}{2 \pi c} \Delta \omega(t)
$$

as a function of time for each component (dashed lines) as well as for the total field (solid line). For each component, $\Delta \lambda(t)$ is obtained by taking the derivative of $\Phi_{x}$ and $\Phi_{y}$ with respect to time [see Eq. (1)]. For the total field, $\Delta \lambda(t)$ is determined numerically by examining the variation in time of $\operatorname{Re}[\mathbf{E}(\mathbf{t})]$. More precisely, we calculate the time interval between the successive extrema of the modulus square of the total field vector $\left[E_{x} \cos \left(\omega_{0} t-\Phi_{x}\right), E_{y} \cos \left(\omega_{0} t-\Phi_{y}\right)\right]$, where $E_{x}$ and $E_{y}$ are the amplitudes of the components in the $x$ and $y$ directions. This time interval $T_{1 / 4}(t)$ can be written as

$$
T_{1 / 4}(t)=\frac{\pi}{2 \omega_{0}}-\Delta T_{1 / 4}(t)
$$
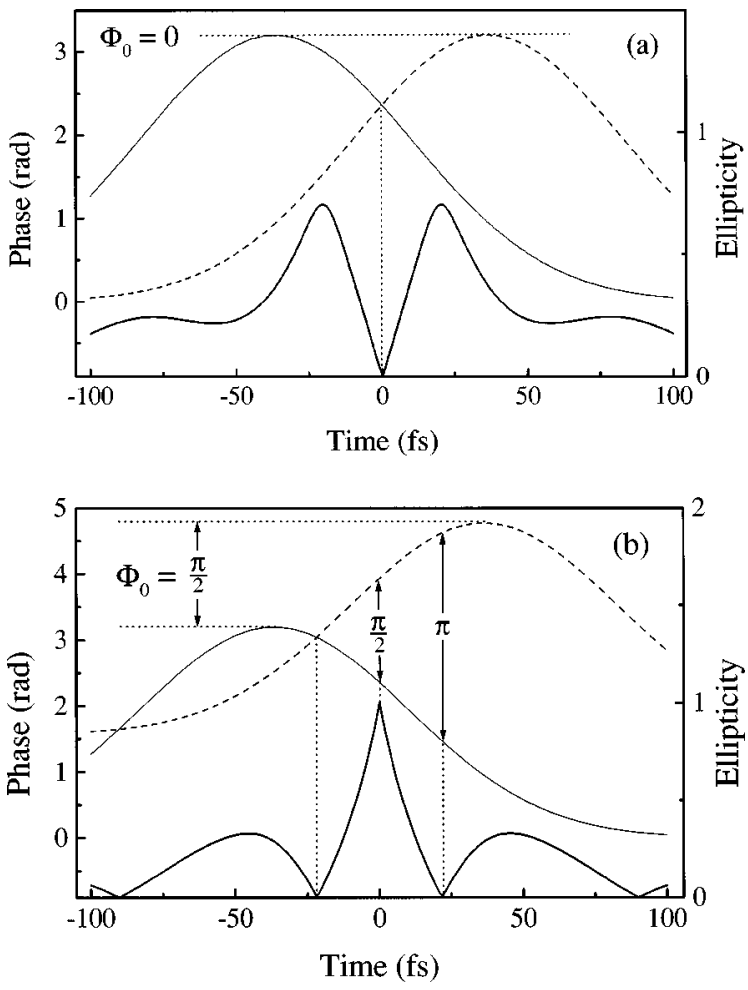

FIG. 2. Phase versus time for the two orthogonally polarized components delayed by $73 \mathrm{fs}$ (thin solid and dashed lines). The laser ellipticity versus time is shown as a thick line at the bottom. The laser pulse duration before the quartz plate is $110 \mathrm{fs}$. In (a) the phase difference is 0 in the absence of chirp, whereas in (b) it is $\pi / 2$. The $B$ integral is 3 .

with $\Delta \lambda(t)=4 c \Delta T_{1 / 4}(t)$. The calculation fails when the field is circularly polarized, because the modulus of electric field vector becomes time independent. This happens around $t=0$ in the case $\Phi_{0}=\pi / 2$, as indicated by the (short) dashed line in Fig. 3, and for $t \approx \pm 20 \mathrm{fs}$ in the case in the case $\Phi_{0}$ $=0$. Apart from these region(s) where the calculation fails, the chirp obtained does not depend on $\Phi_{0}$, so the total chirp can be obtained by superposing results of calculations performed with different $\Phi_{0}$. As expected, the wavelength shift of the total field follows that of the first component for early times $(t<-30 \mathrm{fs})$ and that of the second component for late

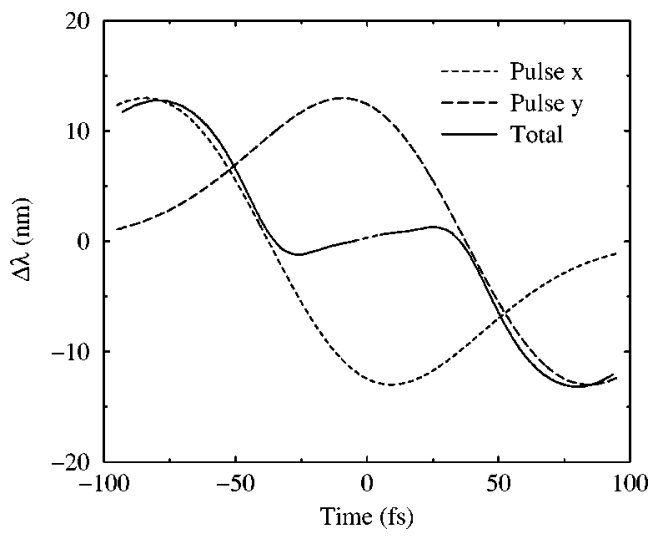

FIG. 3. Wavelength shift vs time $\Delta \lambda(t)$ for the two orthogonally polarized components (dashed lines) and for the total pulse (solid line); $B=3$. 
times $(t>30 \mathrm{fs})$. For $-30 \mathrm{fs}<t<30 \mathrm{fs}$, the wavelength modulation is rather small, about a factor of 10 smaller than that of the components on each axis, and, which is of importance for the interpretation of our experimental results, increasing. This corresponds to a negative frequency chirp for the total field, even though the chirp induced on each of the components is positive.

\section{F. Laser ellipticity}

Since the phase difference between the two perpendicular components varies in time, the total field is elliptically polarized, with a time-varying degree of ellipticity. The instantaneous value of the ellipticity is [22]

$$
\boldsymbol{\epsilon}(t)=\tan \left[\frac{1}{2} \arcsin \left(\frac{2 E_{x} E_{y} \sin \left(\Phi_{x}(t)-\Phi_{y}(t)\right)}{\left|E_{x}\right|^{2}+\left|E_{y}\right|^{2}}\right)\right] .
$$

In Figs. 2(a) and 2(b) (bottom curve, thick line), we plot $|\epsilon(t)|$ for the two choices of $\Phi_{0}, 0$, and $\pi / 2$. In Fig. 2(a), the ellipticity becomes 0 (linear polarization) only once, at $t$ $=0$, and remains otherwise larger than 0.2. In Fig. 2 (b), the ellipticity becomes 0 twice, at $t= \pm 23$ fs. The width of the temporal "gates," during which harmonic emission is possible, can be estimated by assuming, in a phenomenological way, that the harmonic yield behaves as a function of the degree of ellipticity as $\exp \left(-\gamma|\epsilon|^{2}\right)$ with $\gamma \simeq 60[7,22]$. This dependence is the best fit to the experimental variation of the harmonic yield (for orders around 41 and in neon) versus the laser degree of ellipticity. The widths at half maximum of the central gate in Fig. 2(a) as well as of the two gates in Fig. 2(b) are found to be about 5 fs.

It should be stressed that, in our case, in contrast to other schemes [6], the time variation of the ellipticity is nonperiodical, being rapid around the peak of the pulse and much slower in the wings. The rapid variation around the peak of the laser pulse allows us to produce a narrow gate [or two narrow gates in the case of Fig. 2(b)], while the slow variation in the wings allows us to avoid the recurrence of further gates during the laser pulse.

\section{EXPERIMENTAL RESULTS}

The first experiment consists in comparing the harmonic spectra with and without the chirp-inducing glass plate for the two cases $\Phi_{0}=0$ and $\Phi_{0}=\pi / 2$. In the $\Phi_{0}=0$ case, the introduction of the glass plate leads to a decrease of the harmonic signal by about one order of magnitude. This is because the time during which the polarization is linear is considerably reduced for $\Phi_{0}=0$. In the second case, $\Phi_{0}$ $=\pi / 2$, in contrast, the introduction of the glass plate leads to an increase of the signal by about one order of magnitude. The polarization is approximately circular without the glass plate and the harmonic signal is very small. Although harmonic generation is in principle not possible, the very small signal is expected to be due to the additional chirp from the rest of the optics. Adding a chirp ensures that the polarization becomes linear during a short time, leading to harmonic emission.

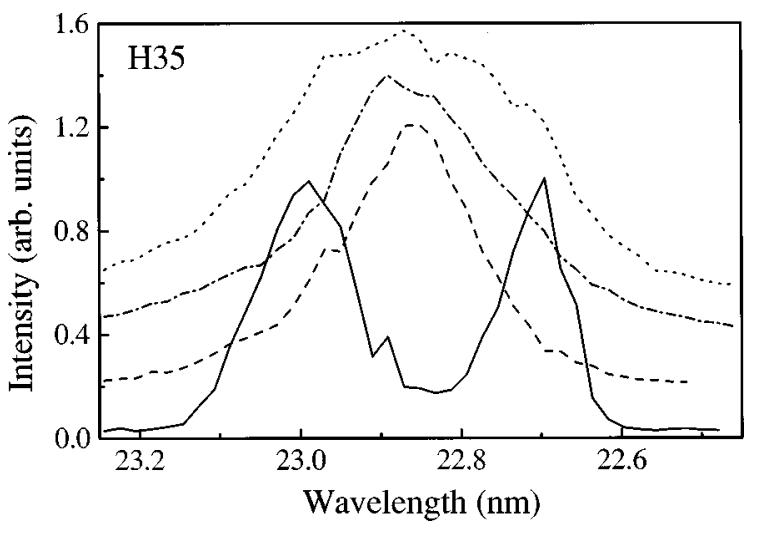

FIG. 4. Normalized 35th harmonic spectrum in four cases: with glass plate inserted and quartz plate axis parallel to the laser field polarization (dotted line), without glass plate and quartz plate axis parallel to the laser field polarization (dot-dashed line), with glass plate, quartz plate oriented at $45^{\circ}$ to the laser polarization, $\Phi_{0}=0$ (dashed line) and $\Phi_{0}=\pi / 2$ (solid line). The laser intensity in the jet is $3 \times 10^{14} \mathrm{~W} / \mathrm{cm}^{2}$ and the intensity on the glass plate is 8 $\times 10^{10} \mathrm{~W} / \mathrm{cm}^{2}$. The four curves have been slightly shifted vertically relative to each other for the sake of clarity.

\section{A. Harmonic spectra}

Typical normalized spectra are shown in Fig. 4 for the 35 th harmonic. The two curves at the top are obtained with the optic axis of the quartz plate parallel to the laser field, i.e., $\alpha=0$, constant ellipticity. The dotted line and the dotdashed lines are obtained with and without the glass plate, respectively. The dashed and solid lines, obtained with a time-varying ellipticity, $\alpha=45^{\circ}$, correspond to the two cases discussed, $\Phi_{0}=0$ and $\Phi_{0}=\pi / 2$, respectively. The intensity is estimated to be $3 \times 10^{14} \mathrm{~W} / \mathrm{cm}^{2}$, and a factor 1.5 higher when $\alpha=0$. The result shown as a dotted line exhibits a broadening, compared to the spectrum obtained in the same conditions, but without the glass plate, owing to self-phase modulation in the glass plate. The full width at half maximum (FWHM) is about $0.35 \mathrm{~nm}$, whereas it is about $0.25 \mathrm{~nm}$ without the glass plate. The result obtained for $\alpha=45^{\circ}, \Phi_{0}$ $=0$ (dashed line) shows a rather regular and narrow peak (FWHM of $0.20 \mathrm{~nm}$ ). The result obtained for $\alpha=45^{\circ}, \Phi_{0}$ $=\pi / 2$ shows two distinct narrow peaks, separated by about $0.3 \mathrm{~nm}$, contained, however, in the bandwidth of the spectrum with the chirp, but without the ellipticity modulation. These peaks, each of width about $0.15 \mathrm{~nm}$, are located symmetrically on either side of the central harmonic wavelength. We checked that the spectrum of the fundamental laser field measured after the quartz plate did not exhibit such structure and did not depend on the rotation angle $\beta$ (and hence on the phase difference $\Phi_{0}$ ). The resolution of our spectrometer is estimated to be below $0.05 \mathrm{~nm}$.

The result obtained for $\Phi_{0}=0$ is a rather counterintuitive one: By limiting the harmonic emission to a short time (a few femtoseconds), we obtain a narrower frequency spectrum than with linear polarization (with or without the glass plate). However, this simply means that the harmonics of a linearly polarized femtosecond laser are not Fouriertransform limited. They exhibit a frequency chirp, as predicted theoretically $[8,10]$. When we restrict the harmonic emission to a short time, we "select" the part of the harmonic spectral content that is emitted during that short time, 


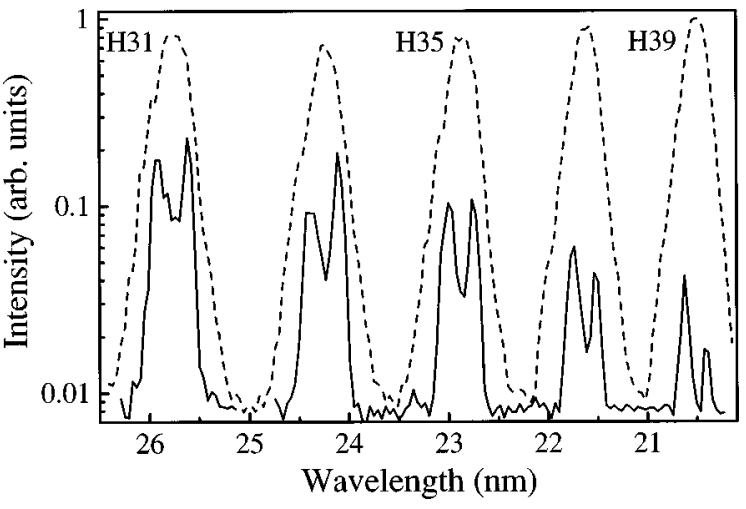

FIG. 5. Harmonic spectra in two cases: with glass plate inserted and quartz plate axis parallel to the laser field polarization (dotted line), and with glass plate, quartz plate oriented at $45^{\circ}$ to the laser polarization and $\Phi_{0}=\pi / 2$ (solid line). The laser intensity in the gas jet is $3 \times 10^{14} \mathrm{~W} / \mathrm{cm}^{2}$ and the intensity on the glass plate is $7 \times 10^{10} \mathrm{~W} / \mathrm{cm}^{2}$

obtaining a narrow frequency spectrum. The Fourier transform of a spectrum for the 35th harmonic with a $0.2-\mathrm{nm}$ bandwidth, if we assume the chirp to be negligible over the short duration of the gate, is a $4 \mathrm{fs}$ pulse. This duration is comparable to the estimated 5 fs gate width.

The result obtained for $\Phi_{0}=\pi / 2$ confirms this interpretation. The double peak structure can be simply interpreted as the consequence of the frequency modulation of the harmonic field. When we restrict the emission in time to (essentially) two gates, we select two frequency windows. If there were no chirp, the spectrum of two pulses delayed in time, but with the same frequency content, would exhibit interferences. This is not observed in the present experiment. Instead, two distinct frequency peaks are observed. Similar results are obtained for all of the harmonics in the plateau. Figure 5 shows, for example, the result obtained for $\alpha=0$, compared to that obtained in the $\alpha=45^{\circ}, \Phi_{0}=\pi / 2$ case. The harmonic spectra obtained in the latter case show a clear double-peak structure, with a separation increasing with the wavelength. In the rest of this section, we concentrate on the $\Phi_{0}=\pi / 2$ case, and study more systematically how the peak separation depends on the different experimental parameters.

\section{B. Study of the double-peak structure}

Figure 6 shows the influence of the laser intensity in the interaction region on the peak separation in the $\Phi_{0}=\pi / 2$ case, for the 35th harmonic (the experimental results are shown as solid squares). The laser energy, hence the amount of laser-induced chirp, is kept constant, and the focused intensity is simply varied by changing the diameter of the aperture from 14.5 to $18 \mathrm{~mm}$, corresponding to intensities in the gas jet from 2.3 to $5.4 \times 10^{14} \mathrm{~W} / \mathrm{cm}^{2}$. The peak separation increases with the intensity, which indicates that the harmonic chirp originates, at least partly, from the harmonic generation process in the nonlinear medium.

In Fig. 7, we show how the frequency separation depends on the harmonic order. From the 23rd to the 41st harmonics, the peak separation increases by less than 50\%. Finally, in Fig. 8, we present normalized harmonic spectra obtained at different laser energies, corresponding to intensities on the glass plate varying from 6.2 to $7.7 \times 10^{10} \mathrm{~W} / \mathrm{cm}^{2}$.

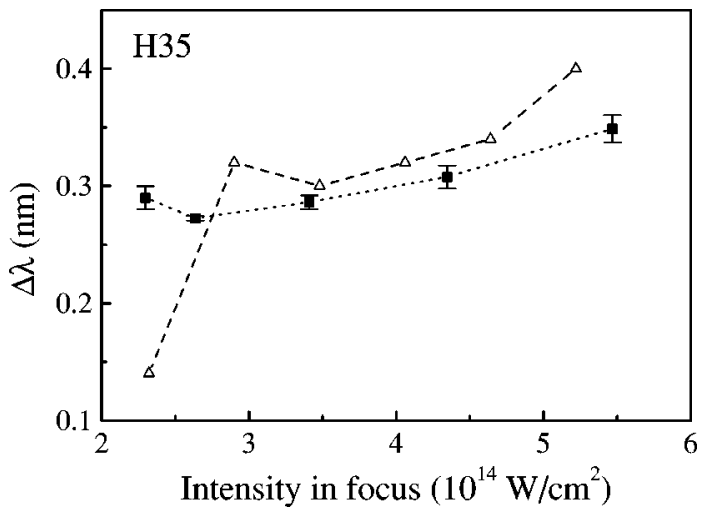

FIG. 6. Wavelength separation for the 35th harmonic (squares) as a function of the intensity in the interaction region. The intensity on the glass plate is $7.4 \times 10^{10} \mathrm{~W} / \mathrm{cm}^{2}$. The open triangles refer to theoretical results obtained in the same conditions $(B=3)$.

\section{DISCUSSION}

\section{A. Different contributions to the harmonic chirp}

To interpret the experimental results presented in the previous section, we discuss in turn the different contributions to the harmonic phase modulation.

\section{Laser chirp}

The harmonics are expected to exhibit some amount of chirp, simply because the laser field used to create the harmonic radiation is frequency chirped. For $B=3$, as shown in Fig. 3, and for -30 fs $<t<30$ fs, the laser wavelength modulation $\Delta \lambda / \Delta t$ is approximately linear, and equal to $0.044 \mathrm{~nm} / \mathrm{fs}$. This leads to a harmonic wavelength modulation equal to $\Delta \lambda / q \Delta t, q$ denoting the harmonic order. For the 35 th harmonic, we find $\Delta \lambda / q \Delta t=1.3 \times 10^{-3} \mathrm{~nm} / \mathrm{fs}$, i.e., $0.06 \mathrm{~nm}$ for $\Delta t=46 \mathrm{fs}$ (time difference between the two gates). This wavelength separation is smaller than that observed in the experiment. Moreover, it is predicted to be independent of the intensity in the interaction region, for a constant chirp, and inversely proportional to the harmonic order (the frequency separation being proportional to the harmonic order).

\section{Atomic chirp}

Theoretical calculations $[9,12]$ predict that the harmonic phase varies rapidly, and, with our sign convention, de-

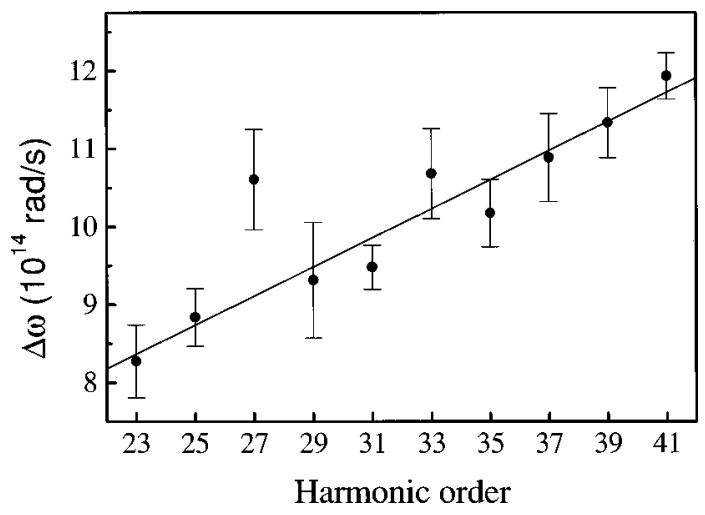

FIG. 7. Frequency separation as a function of harmonic order. The intensity in the jet is $3.4 \times 10^{14} \mathrm{~W} / \mathrm{cm}^{2}$ and the intensity on the glass plate is $7.4 \times 10^{10} \mathrm{~W} / \mathrm{cm}^{2}$. 


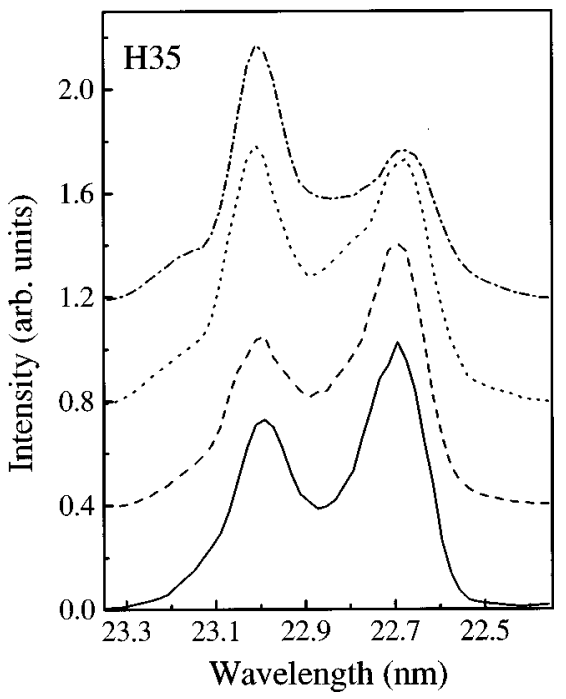

FIG. 8. Normalized 35th harmonic spectra for different $B$ integrals. The four curves have been slightly shifted vertically relative to each other for sake of clarity. From the top to the bottom, the intensity on the glass plate is $7.7,6.9,6.5$, and $6.2 \times 10^{10} \mathrm{~W} / \mathrm{cm}^{2}$, leading to laser intensities in the gas jet between 4.5 and 5.6 $\times 10^{14} \mathrm{~W} / \mathrm{cm}^{2}$.

creases almost linearly with intensity. The variation of the laser intensity during the pulse leads to a significant phase modulation of the harmonic pulse $[8,10,13]$, and consequently to a negative chirp. Using the strong-field approximation [2], this (atomic) phase variation with intensity $(I)$, for a linearly polarized field, can be expressed approximately as $-x I$, where $x=28 \times 10^{-14}$ (with $I$ in $\mathrm{W} / \mathrm{cm}^{2}$ ) for the harmonics in the plateau and $x=13 \times 10^{-14}$ for the harmonics in the cutoff region [10]. Using the value for the plateau region, and at a peak intensity $I=3 \times 10^{14} \mathrm{~W} / \mathrm{cm}^{2}$ (see Fig. 4), the wavelength modulation $\Delta \lambda / \Delta t$, owing to the atomic phase, can be estimated to be $5.7 \times 10^{-3} \mathrm{~nm} / \mathrm{fs}$, i.e., $0.25 \mathrm{~nm}$ for $\Delta t=46$ fs at the 35 th harmonic frequency. The peak separation induced by the atomic chirp alone is predicted to be proportional to the peak intensity in the interaction region. In addition, since the phase variation is, to a large extent, independent of the process order (as long as the harmonics belong to the plateau region), the peak separation in frequency should be relatively independent of the process order.

\section{Ionization chirp}

Finally, ionization, which is inherently present in the harmonic generation process, also leads to a chirp, depending on the density of free electrons, and hence on the gas pressure used in the experiment. We can give an upper estimate of the wavelength shift, over the entire pulse duration, owing to ionization [23]:

$$
\Delta \lambda=-\frac{\lambda^{3} e^{2}}{2 \pi q m c^{3}} \frac{d \mathcal{N}_{e}}{d t} L,
$$

where $e, m$ are the charge and mass of the electron, $\mathcal{N}_{e}$ is the electron density, and $L$ the length of the medium. At saturation, (i.e., an intensity higher than those utilized in the present work), $d \mathcal{N}_{e} / d t \approx \mathcal{N}_{0} / \Delta t$, where $\Delta t$ is the pulse duration and $\mathcal{N}_{0}$ the neutral atom density. In our experimental conditions (with about 20 mbar pressure), we obtain $2.4 \mathrm{~nm}$ for the fundamental, i.e., $0.07 \mathrm{~nm}$ for the 35 th harmonic. Since we are below saturation, and since the time difference considered is much less than the pulse duration, we believe that the ionization chirp (of the same sign as those considered above) does not contribute significantly to the peak separation observed in the present work.

From this discussion, we conclude that the wavelength separation between the two peaks in each harmonic is mainly due to the atomic chirp, with some contribution from the laser chirp. Both chirps are of the same sign. For the 35th harmonic considered in Fig. 4, the sum of the contributions from the atomic and laser-induced chirp gives about $0.3 \mathrm{~nm}$, which corresponds very well to the value measured experimentally. The results presented in Fig. 6 and in Fig. 7 are consistent with the interpretation that the peak separation is due to both contributions: atomic chirp and laser chirp, with the same sign. The peak separation increases approximately linearly with the laser intensity in the interaction region, but with a positive offset. The peak separation in frequency, which should be constant for the atomic chirp and proportional to $q$ for the laser chirp, increases with harmonic order, but not as rapidly as what would be expected if due to the laser chirp only. We find approximately a straight line increasing with $q$, but with a positive offset.

Finally, the result presented in Fig. 8 can be understood as follows: as $B$ increases (linearly with the laser energy), the atomic and laser chirp increase, but the time difference between the two gates decreases (see Fig. 2). The two effects compensate each other and the peak separation becomes practically independent of $B$.

\section{B. Theoretical simulation of the single atom response}

To confirm our interpretation, we have performed a calculation of the harmonic emission from an atom exposed to a laser pulse with the same characteristics as in the experiment. To describe harmonic generation of a laser pulse with frequency and polarization modulation in time is a rather formidable problem. We have therefore performed a simplified calculation, based on the single-atom response only, and using an adiabatic approximation. The theoretical model is based on the strong field approximation [2]. The calculation consists in several steps:

(i) A laser field as used in the present experiment can be characterized by four time-dependent quantities: the ellipticity $\epsilon(t)$ [see Eq. (5)], the intensity $I(t)=|E(t)|^{2}$, the angle of rotation of the polarization ellipse relative to the $(x, y)$ axes, defined as

$$
\chi(t)=\frac{1}{2} \arctan \left(\frac{2 E_{x} E_{y} \cos \left[\Phi_{x}(t)-\Phi_{y}(t)\right]}{\left|E_{x}\right|^{2}-\left|E_{y}\right|^{2}}\right),
$$

and, finally, the frequency $\omega(t)=\omega_{0}+\Delta \omega(t)$ (see Fig. 3).

(ii) We first evaluate the dipole moment $\mathbf{d}_{35}\left(\epsilon_{i}, I_{k}\right)$ for the 35 th harmonic generated in a neon atom, for a number of laser intensities $\left(800\right.$ points between $7 \times 10^{11}$ and 6 $\times 10^{14} \mathrm{~W} / \mathrm{cm}^{2}$ ) and degrees of ellipticity (16 points between 0 and 0.3), as described in Ref. [22]. This is a timeindependent calculation, where the atom is assumed to experience constant intensity and degree of ellipticity. In addi- 
tion, the laser field is supposed to have a constant frequency (no chirp) and the axes of the ellipse coincide with the $(x, y)$ axes.

(iii) To obtain the harmonic field emitted by a single atom at a given time $t$ during the pulse, we first perform a (bilinear) interpolation of the calculated dipoles $\mathbf{d}_{35}\left(\epsilon_{i}, I_{k}\right)$ to get $\mathbf{d}_{35}(\epsilon(t), I(t))$. We thus obtain the response of a $\mathrm{Ne}$ atom to a laser field characterized by $\epsilon(t), I(t), \chi(t)=0, \omega(t)=\omega_{0}$.

(iv) We rotate the axes of the vector $\mathbf{d}_{35}(\epsilon(t), I(t))$ by the angle $-\chi(t)$, in order to obtain the response of an atom to a laser field characterized by $\epsilon(t), I(t), \chi(t), \omega(t)=\omega_{0}$.

(v) We account for the laser chirp in an approximate way, by replacing the $e^{-i 35 \omega_{0} t}$ time-varying factor for a harmonic field with constant frequency (the other time-dependent contributions being assumed to be much more slowly varying), by the factor $\exp \left[-i \int^{t}\left(35 \omega\left(t^{\prime}\right) d t^{\prime}\right)\right]$. This means that we replace the frequency $\omega_{0}$ by the value of the frequency at each time $\omega(t)$, in the rapidly time varying oscillating term. We assume that the (small) change of frequency in time does not affect the other (slow) time-dependent contributions to the electric field. Numerically, we simply multiply the field obtained as outlined above by the phase factor $\exp \left[-i \int^{t} 35\left(\omega\left(t^{\prime}\right)-\omega_{0}\right) d t^{\prime}\right]$. This assumes of course that the phase variation is much slower than that of the harmonic cycle.

(vi) Finally, the power spectrum is obtained by taking the square of the Fourier transform of the harmonic field obtained.

The result obtained for the 35th harmonic in the $\Phi_{0}$ $=\pi / 2$ case are shown in Fig. 9. The solid line indicates the complete calculation, whereas the dashed line shows the calculation performed without any laser chirp. The calculation has been performed at a fundamental wavelength of $794 \mathrm{~nm}$, which explains the difference in wavelength between the experiment and theory. The double-peak structure observed experimentally is very well reproduced in this calculation. We have also calculated how the peak separation increases with intensity for the 35th harmonic. The results are indicated in open triangles in Fig. 6.

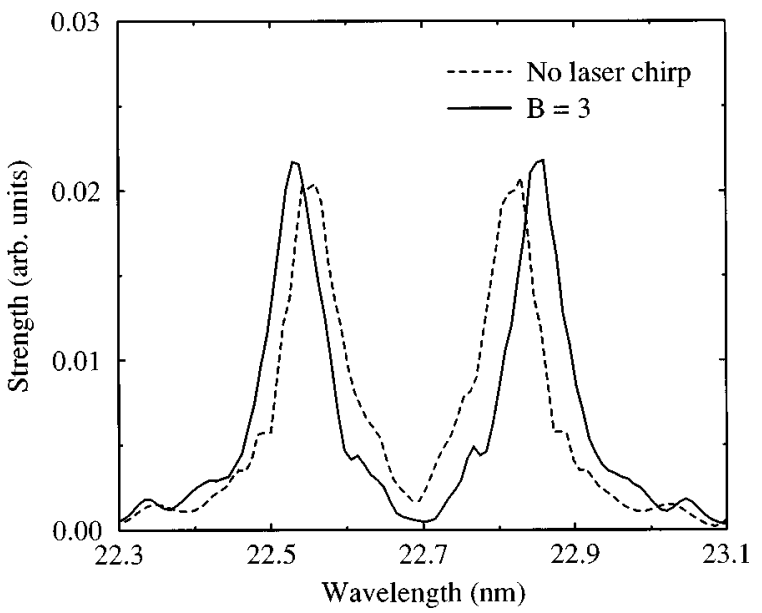

FIG. 9. Theoretical spectra for the 35th harmonic for the $\Phi_{0}=\pi / 2$ case, with (solid line) and without (dashed line) laser chirp. $B=3$ and $I=3.8 \times 10^{14} \mathrm{~W} / \mathrm{cm}^{2}$.

\section{Summary}

In summary, we have manipulated in time the harmonic emission, using birefringent optics and chirped laser pulses. The results show that the harmonic pulses are significantly frequency chirped. By allowing harmonic emission only during a short time, we observe a narrowing of the spectrum. By allowing harmonic emission twice during the laser pulse, we observe a splitting of the harmonic spectra. This splitting is a direct measurement of the harmonic chirp, which is found to be mainly due to the harmonic generation process.

\section{ACKNOWLEDGMENTS}

The support from the Swedish Natural Science Research Council is acknowledged. Discussions with D. Lappas and M. Lewenstein are gratefully acknowledged. C.A. was supported by the European Community, within the Training and Mobility of Researchers Program.
[1] P. B. Corkum, Phys. Rev. Lett. 71, 1994 (1993); K. J. Schafer, B. Yang, L. F. DiMauro, and K. C. Kulander, ibid. 70, 1599 (1993).

[2] M. Lewenstein, Ph. Balcou, M. Yu. Ivanov, A. L'Huillier, and P. Corkum, Phys. Rev. A 49, 2117 (1994).

[3] W. Becker, S. Long, and J. K. McIver, Phys. Rev. A 50, 1540 (1995).

[4] P. Dietrich, N. H. Burnett, M. Yu. Ivanov, and P. B. Corkum, Phys. Rev. A 50, R3585 (1995); K. S. Budil, P. Salières, A. L'Huillier, T. Ditmire, and M. D. Perry, ibid. 48, R3437 (1993); Y. Liang, M. V. Ammosov, and S. L. Chin, J. Phys. B 27, 1296 (1994).

[5] I. Mercer, E. Mevel, R. Zerne, A. L'Huillier, P. Antoine, and C.-G. Wahlström, Phys. Rev. Lett. 77, 1731 (1996).

[6] P. B. Corkum, N. H. Burnett, and M. Y. Ivanov, Opt. Lett. 19, 1870 (1994); M. Y. Ivanov, P. B. Corkum, T. Zuo, and A. Bandrauk, Phys. Rev. Lett. 74, 2933 (1995).
[7] E. Constant, V. D. Taranukhin, A. Stolow, and P. B. Corkum, Phys. Rev. A 56, 3870 (1997).

[8] C. Kan, C. E. Capjak, R. Rankin, and N. H. Burnett, Phys. Rev. A 52, R4336 (1995); C. Kan, C. E. Capjak, and R. Rankin, ibid. 54, R1026 (1996).

[9] M. Lewenstein, P. Salières, and A. L'Huillier, Phys. Rev. A 52, 4747 (1995); P. Salières, A. L'Huillier, and M. Lewenstein, Phys. Rev. Lett. 74, 3776 (1995).

[10] P. Salières, P. Antoine, A. L'Huillier, and M. Lewenstein, Adv. At., Mol., Opt. Phys. (to be published).

[11] G. Farkas and C. Toth, Phys. Lett. A 168, 447 (1992); S. E. Harris, J. J. Macklin, and T. W. Hänsch, Opt. Commun. 100, 487 (1993).

[12] P. Antoine, A. L'Huillier, and M. Lewenstein, Phys. Rev. Lett. 77, 1234 (1996)

[13] K. J. Schafer and K. C. Kulander, Phys. Rev. Lett. 78, 638 (1997). 
[14] M. Protopapas, D. G. Lappas, C. H. Keitel, and P. L. Knight, Phys. Rev. A 53, R2933 (1996).

[15] Ch. Spielmann, N. H. Burnett, S. Sartania, R. Koppitsh, M. Schnürer, C. Kan, M. Lenzner, P. Wobrauschek, and F. Krausz, Science 278, 661 (1997).

[16] I. P. Christov, M. M. Murnane, and H. C. Kapteyn, Phys. Rev. A 57, R2285 (1998); Phys. Rev. Lett. 77, 1743 (1996).

[17] P. Antoine, D. B. Milosevic, A. L'Huillier, M. B. Gaarde, P. Salières, and M. Lewenstein, Phys. Rev. A 56, 4960 (1997).

[18] D. G. Lappas and A. L'Huillier, Phys. Rev. A 58, 4140 (1998).

[19] K. W. DeLong, D. N. Fittinghoff, and R. Trebino, IEEE J.
Quantum Electron. 7, 1253 (1996); R. Trebino and D. J. Kane, J. Opt. Soc. Am. A 10, 1101 (1993).

[20] S. Svanberg, J. Larsson, A. Persson, and C.-G. Wahlström, Phys. Scr. 49, 187 (1994).

[21] G. P. Agrawal, Nonlinear Fibre Optics (Academic Press, Boston, 1989).

[22] P. Antoine, A. L'Huillier, M. Lewenstein, P. Salières, and B. Carré, Phys. Rev. A 53, 1725 (1996).

[23] C.-G. Wahlström, J. Larsson, A. Persson, T. Starczewski, S. Svanberg, P. Salières, Ph. Balcou, and A. L'Huillier, Phys. Rev. A 48, 4709 (1993). 